\title{
Satisfação de Usuários de Próteses Auditivas, com Perda Auditiva de Graus Severo e Profundo
}

\section{Satisfaction of Hearing Aids Users With Hearing Loss of Severe and Deep Degree}

\author{
Alexandre Hundertmarck Lessa*, Maristela Julio Costa**, Karine Thais Becker***, \\ Ana Valéria Almeida Vaucher****.
}

\footnotetext{
* Fonoaudiólogo. Mestrando em Distúrbios da Comunicação Humana pela Universidade Federal de Santa Maria. Pós-graduando em nível de mestrado. Bolsista CAPES. ** Fonoaudióloga. Doutora em Ciências dos Distúrbios da Comunicação Humana pela Universidade Federal de São Paulo. Professora Adjunta do Departamento de Fonoaudiologia da Universidade Federal de Santa Maria.

**** Fonoaudióloga. Mestranda em Distúrbios da Comunicação Humana pela Universidade Federal de Santa Maria. Pós-graduanda em nível de mestrado.

***** Fonoaudióloga. Mestre em Linguística Aplicada pela Pontifícia Universidade Católica do Rio Grande do Sul.

Instituição: Universidade Federal de Santa Maria.

Santa Maria / RS - Brasil.

Endereço para correspondência: Alexandre Hundertmarck Lessa - Rua Conde de Porto Alegre, 961, Apto. 801 - Centro - Santa Maria / RS - Brasil - CEP: $97015-110$ - Telefone: (+55 55) 8432-9337 - E-mail: alexandrehl@gmail.com

Bolsa Fundo de Incentivo à Extensão (FIEX-UFSM).

Artigo recebido em 26 de Maio de 2010. Artigo aprovado em 20 de Junho de 2010.
}

\section{RESUMO}

Introdução:

Objetivo:

Método:

Resultados:

Conclusão:

Palavras-chave:

\section{SUMMARY}

Introduction:

Objective:

Method:

Results:

Conclusion:

Keywords:
É necessário analisar a eficácia da protetização, considerando a necessidade de amplificação e dificuldade na comunicação.

Analisar a satisfação de adultos e idosos, usuários de próteses auditivas, com perda auditiva de graus severo e profundo, usando um questionário e pesquisar fatores que possam dificultar a adaptação.

Estudo retrospectivo de 56 pacientes, com idades entre 18 e 86 anos, perda auditiva de graus severo e profundo e protetizados por um programa de concessão de próteses auditivas, submetidos ao questionário [i]Satisfaction with Amplification in Daily Life[/i]. Foram calculadas as médias global, por subescala e por questão e deu-se ênfase às questões que resultaram em melhores e piores médias.

$58,9 \%$ mulheres e $41,1 \%$ homens, cerca de $55 \%$ dos pacientes apresentaram perda auditiva de grau severo e $45 \%$ profundo. $87,5 \%$ referiu uso maior que 8 horas diárias. Os usuários acreditam que a aquisição da prótese auditiva foi a melhor opção e que esta auxilia muito no entendimento da fala. Sentem-se mais confiantes e capazes com a amplificação sonora e referem bom atendimento pelos profissionais do serviço. O desempenho ao telefone foi o problema encontrado.

Foi demonstrado elevado grau de satisfação na população estudada. O grau da perda auditiva, assim como a incompatibilidade de sistemas entre telefone e bobina telefônica e a dificuldade no posicionamento do telefone são fatores que dificultam o bom desempenho ao telefone. A acentuada perda auditiva colabora para que a prótese auditiva seja parte integrante do dia-a-dia e essencial no auxílio à comunicação. ACTRN12610000424000 saúde pública, perda auditiva, auxiliares de audição, satisfação do paciente, adulto, idoso.

It is necessary to analyze the efficiency of the hearing aids use, considering the necessity of enlargement and communication difficulty.

To analyze the satisfaction of adults and elders, users of hearing aids with hearing loss of severe and deep degree, using a questionnaire and searching factors that can difficult the adaptation.

A retrospective study of 56 patients, with age group between 18 and 86 years old, hearing loss of severe and deep degree and users of HA by a program of concession of hearing aids, submitted to the questionnaire: "Satisfaction with Amplification in Daily Life". The global average was calculated by sub scale and by question and it was given emphasis to the questions that resulted in best and worse averages.

$58,9 \%$ were women and $41,1 \%$ men, about of $55 \%$ of the patients presented hearing loss of severe degree and $45 \%$ deep degree. $87,5 \%$ referred use major than 8 hours daily. The users believe that the acquisition of the hearing aid was the best option and this aid very much in the speech understanding. They feel themselves trustful and able with the sounding amplification and refer a good service by professionals of the service. The performance to the telephone was the problem found.

It was demonstrated high degree of satisfaction in the studied population. The hearing loss degree, as well as the incompatibility of systems between telephone and telecoils and the difficulty in the placement of telephone ate factors that difficult the good performance to the telephone. The accentuated hearing loss collaborate for the hearing aid be an integral part of the daily life and essential in the aid to the communication. ACTRN12610000424000

public health, hearing loss, hearing aids, patients satisfaction, adult, elders. 


\section{INTRODUÇÃO}

A deficiência auditiva é considerada a terceira incapacidade mais comum na população (1). Ela causa no indivíduo, além da dificuldade para ouvir, comprometimentos psicossociais, já que pode haver afastamento do convívio social e das atividades ocupacionais, acometendo a qualidade de vida do mesmo.

Uma das formas de diminuir o impacto da perda auditiva na vida de um indivíduo é através do uso de próteses auditivas. Assim, os sons ambientais e de fala serão amplificados, além de sinais de perigo e alerta (2).

Em 2004, considerando as condições de acesso da população brasileira aos procedimentos de saúde auditiva, o Ministério da Saúde instituiu a Política Nacional de Atenção à Saúde Auditiva, através da Portaria no 2.073 (3) de 28 de setembro de 2004, que garante desde o diagnóstico até a protetização de usuários do Sistema Único de Saúde.

No ano de 2006, a Universidade Federal de Santa Maria assinou o convênio com o Ministério da Saúde e passou a realizar a concessão de próteses auditivas, de acordo com a Política citada.

Pelo fato de ser um amplificador sonoro, a prótese auditiva necessita de uma reserva coclear suficiente para que possa haver uma boa percepção do som e da fala pelo paciente. Alguns indivíduos, porém, apresentam uma disfunção auditiva tão importante que mesmo uma prótese auditiva potente não consegue ajudá-los (4).

Em alguns casos, curiosamente, observamos que existem pacientes que, apesar de apresentarem benefício com o aparelho, não se encontram muito satisfeitos. Em contrapartida, outros, mesmo sem grande benefício, demonstram grande satisfação em serem usuários de aparelho de amplificação sonora individual - AASI (5).

A experiência clínica mostrou ao fonoaudiólogo que a relação entre sistemas de amplificação e necessidades acústicas encontradas nos exames clínicos não garantem a efetividade da adaptação do usuário ao equipamento. Foi necessário que o fonoaudiólogo enfocasse sua avaliação no sujeito e não mais na deficiência auditiva, para encontrar uma prática clínica mais satisfatória (6).

Testes objetivos, como ganho funcional e inteligibilidade de fala não bastam para sabermos o quanto foi efetiva a adaptação, já que mesmo que a prótese auditiva forneça uma boa audibilidade, o paciente poderá não estar satisfeito se ainda assim não houver redução das dificuldades auditivas e desvantagens psicossociais (7-8).

Assim, há um interesse crescente no desenvolvimento de procedimentos de validação que permitam avaliar o benefício do usuário fora do ambiente clínico, constituindo-se em questionários de auto-avaliação (9).

O questionário Satisfaction With Amplification In Daily Life-SADL (10) foi desenvolvido com a intenção de prover não apenas um índice de satisfação global, mas também um perfil que possa ser utilizado para identificar as áreas dos problemas que causam a insatisfação (11).

Diversos estudos (10,12-16) que utilizaram o questionário SADL mostraram que os pacientes estavam consideravelmente satisfeitos, mas identificaram também em quais aspectos houve menor satisfação.

Em um destes (15), o autor hipotetiza que o fato de 90\% dos indivíduos de sua amostra apresentar média tonal de grau normal, leve ou moderado tenha favorecido os bons resultados de satisfação obtidos, visto que acredita que a performance do AASI seja pior em casos de perdas auditivas mais graves.

Essa hipótese instigou à investigação da satisfação nos casos em que as perdas auditivas são bastante acentuadas. Assim, a população avaliada pelo presente estudo apresenta a importante particularidade de ser composta justamente por estes indivíduos. Dessa forma, analisamos a eficácia da protetização nestes usuários, considerando dentre outros fatores, a grande necessidade de amplificação e a dificuldade na comunicação dos mesmos.

Além disso, na rotina de atendimentos realizados no programa de concessão de próteses auditivas, após o término das consultas obrigatórias, em muitos casos o serviço perde o contato com os pacientes. Esse fato pode conduzir à desistência do uso dos aparelhos auditivos, além da possibilidade de conservação inadequada dos mesmos, devido à ausência do reforço das informações quanto ao uso, manuseio e manutenção adequados.

A falta de acompanhamento dos deficientes auditivos traz consequências negativas para os mesmos e para a sociedade. O uso não efetivo da prótese compromete a integração social, incluindo o meio educacional e ocupacional.

Devido ao custo despendido pelos serviços de saúde que fornecem próteses auditivas, à complexidade da adaptação à amplificação sonora e à importância do sucesso da reabilitação aural na vida do portador de perda auditiva, é de extrema relevância o acompanhamento do 
processo de adaptação. Tal monitoramento deve objetivar a avaliação dos procedimentos clínicos e a garantia da qualidade dos serviços, além de analisar a satisfação dos usuários com os aparelhos que lhe foram disponibilizados, pois tais ações refletem a realidade dos resultados alcançados com o programa de concessão.

Contudo, o objetivo deste estudo é verificar a satisfação com as próteses auditivas de usuários adultos e idosos com perdas auditivas de graus severo a profundo, atendidos pelo programa de concessão de próteses auditivas de fluxo contínuo da Secretaria de Assistência à Saúde do Ministério da Saúde, desenvolvido na Universidade Federal de Santa Maria e pesquisar os fatores que possam dificultar a adaptação efetiva das próteses auditivas nessa população.

\section{MétOdo}

O estudo foi realizado no Laboratório de Próteses Auditivas do Serviço de Atendimento Fonoaudiológico (SAF) da Universidade Federal de Santa Maria (UFSM). A coleta dos dados se deu no período compreendido entre maio e agosto de 2009.

Esta pesquisa é um subprojeto dentro de um projeto maior intitulado: Pesquisa e Base de Dados em Saúde Auditiva, registrado no Gabinete de Projetos sob o $n^{\circ}$ 019731 e aprovado pelo Comitê de Ética em Pesquisa com certificado de $\mathrm{n}^{\mathrm{o}}$ 0138.0.243.246-06, em 05/12/2006.

Foram selecionados usuários de próteses auditivas, com idade superior a 18 anos; perda auditiva de grau severo a profundo em ambas as orelhas; protetizados no Laboratório de Próteses Auditivas da UFSM por meio do credenciamento celebrado entre a Secretaria de Saúde do Estado do Rio Grande do Sul e a Universidade Federal de Santa Maria, a partir de 2005, com base nas Portarias 587 e 589 (3), da Secretaria de Assistência à Saúde do Ministério da Saúde, publicadas em outubro de 2004; com tempo mínimo de três meses de uso da amplificação, pois a experiência clínica tem demonstrado ser este um tempo razoável para adaptação à prótese auditiva, sendo possível verificar os reais resultados da intervenção, já que os benefícios advindos do uso da amplificação não emergem de imediato (17).

Tentou-se, através de contato telefônico, a convocação de 166 pacientes que atendiam aos critérios de inclusão. Destes, 60 não foram encontrados, já que não atenderam às ligações, os telefones estavam desatualizados ou os celulares desligados. Os 106 restantes foram questionados quanto à possibilidade de comparecimento ao Laboratório de Próteses Auditivas - 3 haviam falecido, 1 tido os AASI roubados, 2 sido submetidos recentemente à cirurgia, 3 estavam internados no hospital, 5 aguardavam conserto dos aparelhos, 12 haviam consultado há pouco tempo, 8 não poderiam comparecer e afirmaram que entrariam em contato quando possível.

Os 72 indivíduos restantes tiveram a consulta marcada, sendo que 66 compareceram. A estes foram prestados esclarecimentos sobre o objetivo e metodologia da pesquisa e, então, foram submetidos às avaliações após concordarem com a realização dos procedimentos e assinarem o Termo de Consentimento Livre e Esclarecido.

$\mathrm{Na}$ consulta, detectou-se que um dos pacientes, após cirurgia otológica teve melhora dos seus limiares auditivos, tendo a nova configuração da perda auditiva caracterizada como moderadamente severa; 2 pacientes não faziam uso dos aparelhos; 2 apresentaram alterações neurológicas e 5 tiveram os aparelhos mandados para conserto - fatos que interfeririam na coleta de dados e, portanto, foram excluídos do estudo.

Assim, 56 indivíduos, que atenderam a todos os critérios de inclusão, compuseram a amostra.

\section{Procedimentos}

Os pacientes foram submetidos a uma anamnese, que serviu para seleção da amostra e contém questionamentos, principalmente acerca da protetização, do uso efetivo do aparelho auditivo e qualidade do mesmo.

Já a medida da satisfação com o aparelho auditivo na vida diária foi realizada por meio do questionário Satisfaction With Amplification in Daily Life - SADL (10), composto por um total de 15 questões.

Para cada questão, há 7 alternativas, com escala de 1 a 7 pontos atribuídos à resposta, que indica de "nenhum pouco" a "extremamente" satisfeito. Assim, o teste quantifica a satisfação por meio de um escore de quatro subescalas:

A subescala de "Efeitos Positivos" é composta por 6 itens que englobam questões relacionadas à habilidade comunicativa, localização e qualidade sonora, além de abordar questões psicológicas, já a de "Fatores Negativos", por 3 itens relacionados com desempenho em ambiente ruidoso, microfonia e uso do telefone, criados como um "termômetro" dos problemas da adaptação.

Há ainda a subescala de "Serviços e Custos", com 3 itens associados à competência profissional, preço do produto e qualidade do aparelho e a referente à "Imagem Pessoal", na qual estão compreendidos 3 itens relacionados 
com fatores estéticos e o estigma do uso da prótese auditiva.

Considerando que o grupo de amostra é composto por pacientes usuários do Sistema Único de Saúde e que, portanto, tiveram os aparelhos doados, a questão relacionada ao preço do produto não foi aplicada.

Embora a proposta original sugira que o questionário seja respondido pelo próprio paciente, os pesquisadores realizaram leitura em voz alta e anotação das respostas para o instrumento utilizado, com o intuito de minimizar as dificuldades de compreensão das perguntas, relacionadas ao grau da perda auditiva.

\section{Análise dos dados}

A análise dos dados foi feita segundo os valores estabelecidos pelo estudo original (10), que norteou a presente análise.

Assim sendo, para calcular o score global do SADL, realizou-se a média aritmética entre os valores atribuídos às respostas obtidas nas 14 questões aplicadas aos 56 pacientes - sendo 7 a pontuação máxima, que indica maior satisfação. Para o escore de cada subescala, a média aritmética calculada foi realizada com a pontuação referente às respostas dadas às questões que compõem cada subescala.

A fim de melhorar a compreensão dos dados usados, serão apresentados na Tabela 1 os valores encontrados pelos autores do questionário com a interpretação dos seus resultados.

Se os escores ficarem abaixo do $20^{\circ}$ percentil é indicativo de usuários "insatisfeitos", ao passo que entre o $20^{\circ}$ e o $80^{\circ}$ percentil, pacientes estariam "satisfeitos" e acima do valor do $80^{\circ}$ percentil, "muito satisfeitos".

Também foram calculadas as médias aritméticas de todos os indivíduos em cada uma das questões e realizada a análise descritiva dos dados, dando ênfase às questões que resultaram em melhores e piores médias.

\section{RESULTADOS}

Foram avaliados 56 pacientes, sendo 23 homens $(41,1 \%)$ e 33 mulheres $(58,9 \%)$, com idades entre 18 e 86 anos (média de 52,5 anos). Serão apresentados a seguir resultados, em forma de tabelas, mostrando a distribuição dos pacientes, segundo diferentes variáveis. Estas, consideradas a partir dos dados de anamnese e do questionário SADL (Tabelas 2, 3 e 4).
Tabela I. Valores da média, $20^{\circ}$ e $80^{\circ}$ percentil para os escores global e de cada subescala do Satisfaction with Amplification in Daily Life (I0).

\begin{tabular}{lccc}
\hline Escore & Média & $20^{\circ}$ Percentil & $80^{\circ}$ Percentil \\
\hline Global & 4,9 & 4,3 & 5,6 \\
Efeitos Positivos & 4,9 & 3,8 & 6,1 \\
Fatores Negativos & 3,6 & 2,3 & 5,0 \\
Imagem Pessoal & 5,6 & 5,0 & 6,7 \\
Serviços e Custos & 4,7 & 4,0 & 5,7 \\
\hline
\end{tabular}

Tabela 2. Distribuição dos pacientes, segundo a variável grau das perdas auditivas, separados por orelha.

\begin{tabular}{lcccc}
\hline \multirow{2}{*}{ Grau da perda auditiva } & \multicolumn{2}{c}{ Orelha direita } & \multicolumn{2}{c}{ Orelha esquerda } \\
Nevero & $\%$ & N & $\%$ \\
\hline Profundo & 32 & 57,1 & 31 & 55,3 \\
\hline Total & 24 & 42,9 & 25 & 44,7 \\
\hline
\end{tabular}

Tabela 3. Distribuição dos pacientes, segundo a variável tempo de uso diário das próteses auditivas.

\begin{tabular}{lcc}
\hline Horas & $N$ & $\%$ \\
\hline Entre 4 e 8 & 7 & 12,5 \\
Mais que 8 & 49 & 87,5 \\
\hline Total & 56 & 100 \\
\hline
\end{tabular}

Tabela 4. Apresentação do valor médio do Score Global e das médias por subescala e questões referentes do questionário Satisfaction With Amplification in Daily Life (10).

Score Global $\quad 5,77$

Subescala 'Efeitos Positivos' 6, 15

Auxílio dos aparelhos auditivos no entendimento de fala $\quad 6,43$

Convencimento de que a adaptação dos aparelhos

auditivos foi sua melhor opção

Redução da necessidade de repetição para o

entendimento defala

Compensação do problema com o uso dos aparelhos auditivos

Aumento da autoconfiança com o uso dos aparelhos auditivos

Naturalidade do som recebido com o uso dos

aparelhos auditivos

Subescala 'Fatores Negativos'

Frustração com captação de ruídos ambientais

Ocorrência de microfonia com o aumento do volume

Auxílio dos aparelhos auditivos no uso do telefone

Subescala 'Imagem Pessoal'

Percepção da perda auditiva por outras pessoas, decorrente do uso dos aparelhos auditivos

Satisfação com a aparência dos aparelhos auditivos

Sentimento de menosvalia pelo uso dos aparelhos auditivos 6,23

Subescala 'Serviços e Custos' 6,01

Competência dos profissionais do serviço $\quad 6,66$

Satisfação com a qualidade dos aparelhos auditivos $\quad 5,36$ 


\section{DISCUSSÃO}

O gênero predominante dos pacientes avaliados foi o feminino, assim como nos estudos (16,18-19), que pesquisaram a satisfação de usuários de próteses auditivas. De uma forma geral, há um consenso em relação ao maior prejuízo auditivo nos homens do que nas mulheres (20-21), porém há, por parte do homem, em relação à mulher, pouca procura por serviços de saúde (22-24), o que se apresenta como uma justificativa para o maior número de mulheres em estudos que pesquisam a surdez.

A idade dos indivíduos avaliados se mostrou bastante diversificada e bem distribuída, uma vez que a média da idade de todos eles foi bastante aproximada da que resultaria entre a idade mínima e máxima dos testados e que ficaram assim distribuídos: 21 adultos, 15 de meia idade e 20 idosos.

Não se encontra na literatura trabalhos que se proponham a avaliar especificamente a satisfação de usuários de amplificação sonora com perdas auditivas de graus severo e profundo. Nas pesquisas encontradas, a maioria avalia indivíduos com perdas auditivas de graus leve e moderado, algumas incluem os de grau severo e apenas um destes estudos (15) incluiu 2,5\% de pacientes com perda auditiva de grau profundo.

Com relação ao tempo de uso das próteses a maioria dos pacientes avaliados utiliza a amplificação sonora por mais de 8 horas diárias, o que significa que o indivíduo passa a maior parte do dia usando as próteses, ou usa durante os períodos que mais estará sujeito às situações de comunicação. Este é um tempo considerável, pois das 24 horas diárias, aproximadamente 8 horas passamos dormindo, restam 16 horas, das quais, no mínimo em 8 está sendo feito o uso pela maioria, o que demonstra que as próteses auditivas fazem parte integrante do dia-a-dia destes indivíduos.

A média final do score global do SADL ficou em 5,77 , superior às encontradas em pesquisas (12,15-16), que obtiveram valores de 5,05; 5,28 e 5,5 e superior ainda à média e ao $80^{\circ}$ percentil do estudo original (10), revelando pacientes muito satisfeitos.

Um pesquisador (15) afirmou em seu estudo que o alto índice de satisfação dos seus indivíduos poderia ser explicado pelo fato de que a maioria deles apresentava média tonal normal ou de grau leve a moderado e hipotetizou que a performance das próteses auditivas é pior em casos de perdas auditivas mais graves. No entanto, no presente estudo todos os pacientes eram acometidos por perda auditiva de grau severo e profundo e a média de escore global foi superior - 5,77 - ao do estudo referido $-5,28$.

A subescala de Efeitos Positivos teve maior média - 6,15, do que as encontradas em estudos (12,15-16), que pontuaram 4,99; 5,66 e 5,87; e do que a média e o $80^{\circ}$ percentil encontrado na pesquisa original (10). Esta foi a subescala com maior score, e em comparação ao estudo original, demonstra grande satisfação neste importante item que verifica a qualidade sonora e a melhora na comunicação.

Na subescala de Fatores Negativos, outras pesquisas (12,15-16) encontraram médias de 4,5; 4,18 e 5,2 apenas este superior a do presente estudo - 5,08. Como em todas as outras pesquisas, esta foi a subescala com o menor score, já que avalia aspectos considerados problemáticos na adaptação. Ainda assim, os usuários avaliados no presente estudo estão muito satisfeitos, em comparação aos resultados da pesquisa original do SADL (10), tendo obtido média superior ao $80^{\circ}$ percentil. Dois problemas correntes nas pesquisas realizadas com o questionário são: o uso do telefone (confirmado nesta pesquisa) e o incômodo com ruídos ambientais (que possivelmente pelas características auditivas dos pacientes estudados não mostrou influência na queda do score, já que muitas vezes não os escutam e, quando o fazem, não lhes incomoda ou mesmo gostam).

A subescala que avalia a Imagem Pessoal teve média de 5,53, compatível às médias encontradas em outros estudos $(10,12,15-16)$ e indica satisfação para este grupo de questões que abordam o estigma da prótese auditiva.

Já a subescala de Serviços e Custos foi aplicada aos indivíduos de estudos $(12,15)$, que obtiveram médias de 4,94 e 5,61 - inferiores a encontrada no presente estudo - 6,01. Este score é superior ao $80^{\circ}$ percentil da pesquisa original (10) e indica muita satisfação no item. Na avaliação destes aspectos foi excluída a questão referente ao custo da prótese auditiva - já que os indivíduos do estudo foram beneficiados pelo programa de concessão de próteses auditivas - o que possivelmente, em conjunto ao contentamento com o atendimento recebido e a baixa necessidade de consertos, ajudou na elevação desta média.

Algumas questões do teste merecem destaque, pois se sobressaíram na elevação do escore global: a pergunta que aborda se "a aquisição do aparelho foi a melhor opção" teve a maior média - 6,84. Também resultaram em médias altas as questões referentes à competência dos profissionais que atenderam o usuário - 6,66, ao aumento da confiança com a utilização do aparelho auditivo - 6,53, ao 
auxílio que a prótese dá ao usuário no entendimento da fala - 6,43, a não se sentir menos capaz pela necessidade do uso da prótese auditiva - 6,23 e à ausência de microfonia, quando o volume é aumentado - 6,2.

Assim como as citadas anteriormente, que elevaram a média, deve-se dar atenção à questão que diz respeito ao uso do telefone, pois apenas nesta foi evidenciado um baixo escore - 3,61.

A alta média para a questão que aborda se o aparelho auditivo foi a melhor opção para o usuário - 6,84 - traduz o alto nível de satisfação dos indivíduos pesquisados. Nesse caso, o grande comprometimento auditivo pode justamente ser a justificativa para o alto índice, já que durante a aplicação do questionário, comentários como "gosto muito, eu não vivo sem meu aparelho" foram frequentes. Ou seja, estes usuários são tão dependentes da prótese auditiva - e escutam tão pouco sem ela - que o benefício sentido é extremamente grande. Assim, pode ser suposta uma relação entre a satisfação e a dependência do usuário à prótese auditiva.

A questão referente à competência dos profissionais do serviço também obteve um alto escore - 6,66. Ressaltase que o pesquisador que aplicou o questionário não é o mesmo responsável pela protetização do paciente, deixando-o assim à vontade para quaisquer colocações quanto às reais condições de atendimento. Sabemos que os aparelhos auditivos têm um custo bastante elevado e, a possibilidade do portador de deficiência auditiva receber os seus, gratuitamente, pode exercer função neste alto índice. A extrema gratidão demonstrada pelos usuários, além do bom desempenho dos funcionários, parece influenciar no escore e estar intimamente ligada ao fato de terem sido beneficiados pelo programa de concessão de próteses, já que houve diversos relatos, como "eu não quero incomodar" e "muito obrigado, aqui vocês são muito atenciosos, nem parece SUS".

$\mathrm{O}$ alto índice referente ao aumento da confiança com a utilização do aparelho auditivo - 6,53 - mostra o quanto a amplificação sonora é importante para estes pacientes.

Isso pode ser percebido também através da análise do escore elevado - 6,23 - para a questão "Você acha que usar o aparelho faz você se sentir menos capaz?". Os pacientes reagiam com surpresa a este questionamento e respondiam afirmando que "não, pelo contrário, com ele é que eu me sinto mais capaz" ou "sem ele é que eu não escutava e não podia falar com as pessoas". A confiança e o bom desempenho deles na conversação, demonstrados através da elevada satisfação em outros questionamentos, nos faz perceber e entender o quanto realmente se sentem mais capacitados. O indivíduo com perda auditiva, principalmente de graus mais acentuados, geralmente é acometido por comprometimentos psicossociais - utilizar a prótese, conseguir se comunicar e sentir-se inserido/reinserido na sociedade faz com que ele se sinta mais capaz.

A média referente ao auxílio que as próteses dão ao usuário no entendimento da fala - 6,43 - demonstra o quanto elas auxiliam e permitem a compreensão desta. Considerando a perda auditiva dos pacientes avaliados, este resultado remete também à confiança adquirida pelos usuários com o uso das próteses auditivas, já que por mais que, muitas vezes, as próteses não dêem um ganho tão grande a ponto de os usuários escutarem a fala, em associação às pistas visuais, consideram-na um grande auxílio por os deixarem mais confiantes à conversação.

A maioria dos usuários relatou ausência de microfonia, quando o volume é aumentado, o que gerou o elevado escore de 6,2 para a questão que avalia tal aspecto. No entanto, pelo fato de serem acometidos por perdas auditivas de graus severo e profundo, muitas vezes a microfonia ocorre ao aumento do volume - como visto durante as consultas -, mas eles não escutam o sinal sonoro.

Como referido, a pergunta relacionada ao auxílio que a prótese auditiva dá ao usuário no uso do telefone teve um baixo índice - 3,61 -, assim como em pesquisas $(10,12-14,16)$, em que este foi o item de menor satisfação.

Como pesquisadores (12) afirmaram, o uso do telefone é uma situação auditiva, na qual as limitações tecnológicas das próteses ficam evidenciadas.

Nesse caso, é importante ressaltar que em pacientes com perdas auditivas de grau severo e profundo, tem-se como fator agravante a necessidade de grande amplificação sonora, e a perda da pista visual, que é muito utilizada por estes, dificulta ainda mais o uso do telefone.

Além disso, deve-se também ressaltar que, ao aproximar o telefone da prótese, pode ocorrer microfonia. Além do que, de uma maneira geral, mas mais frequentemente nos idosos, há uma dificuldade com relação à colocação do telefone na posição correta próxima ao microfone da prótese, que nos casos de próteses retroauriculares, fica acima da orelha, ou próximo do local onde o sinal da bobina telefônica estará mais ativa; assim como, a limitação de sistemas, que devem ser compatíveis entre o telefone e a bobina telefônica para que a transmissão do sinal se dê de forma satisfatória.

Dessa forma, treino e reorientações, principalmente quanto ao posicionamento do telefone no microfone da prótese auditiva e à melhora no desempenho com o uso da 
bobina telefônica nesta situação, devem ser reforçados a estes pacientes.

\section{CONCLUSÃO}

O presente estudo demonstrou o elevado grau de satisfação dos adultos e idosos, usuários de próteses auditivas, com perda auditiva de graus severo e profundo, protetizados através de um programa de concessão de próteses auditivas.

Mesmo não havendo insatisfação, a problemática percebida foi em relação ao desempenho dos indivíduos em situações do uso do telefone, que, no caso dos avaliados, combina algumas variáveis: grau da perda auditiva, incompatibilidade de sistemas entre telefone e bobina telefônica e dificuldade no posicionamento do telefone para conversação. Portanto, há necessidade de treinamento para sanar esta e outras dificuldades que possam surgir no processo de adaptação.

A acentuada perda auditiva dos avaliados colabora para que a prótese auditiva seja parte integrante do seu diaa-dia e essencial no auxílio à comunicação. Assim, eles acreditam ser esta a melhor opção para reduzir suas dificuldades e estão satisfeitos com a escolha, que os faz sentirem-se mais capazes.

\section{REFERÊNCIAS BIBLIOGRÁFICAS}

1. Instituto Brasileiro de Geografia e Estatística. Censo Demográfico 2000 - Tabulação avançada - resultados preliminares da amostra. Acesso em: 03 de junho de 2009. Disponivel em: http://www.ibge.gov.br/home/presidencia/ noticias/08052002tabulacao.shtm.

2. Campos AHC, Russo ICP, Almeida K. Indicação, Seleção e Adaptação de Próteses Auditivas: Princípios Gerais. In: Almeida K, Iorio MCM. Próteses auditivas: fundamentos teóricos \& aplicações clínicas. $2^{\underline{a}}$ ed. São Paulo: Editora Lovise; 2003. p. 35-54.

3. Portal da Saúde. Acesso em: 20 de junho de 2009. Disponível em: http://www.saude.gov.br/sas/.

4. Bento RF, Brito Neto RV, Castilho AM, Goffi Gomez MA, Giorgi SB, Guedes MC. Resultados auditivos com o implante coclear multicanal em pacientes submetidos à cirurgia no Hospital das Clínicas da Faculdade de Medicina da Universidade de São Paulo. Rev Bras Otorrinolaringol. 2004, 70:632-37.

5. Assayag FHM, Russo ICP. Avaliação subjetiva do benefício e dos efeitos proporcionados pelo uso de amplificação sonora em indivíduos idosos. Rev Dist Comun. 2006, 18(3):38390 .

6. Russo IEP. Intervenção fonoaudiológica para a terceira idade. Rio de Janeiro: Revinter; 1999.

7. Bucuvic EC, Iorio MCM. Próteses auditivas: estudo comparativo das dificuldades auditivas e do benefício da amplificação em pacientes usuários de amplificação não linear e linear. Rev Ciên Méd Biol. 2003, 2(1):77-87.

8. Costa MHP, Sampaio ALL, Oliveira CACP. Avaliação do benefício da prótese auditiva digital e da percepção da desvantagem auditiva ou "handicap" em idosos não institucionalizados. Arq Int Otorrinolaringol. 2007, 11(2):15968.

9. Almeida K. Avaliação dos Resultados da Intervenção. In: Almeida K, Iorio MCM. Próteses auditivas: fundamentos teóricos \& aplicações clínicas. $2^{\underline{a}}$ ed. São Paulo: Editora Lovise; 2003. p. 335-52.

10. Cox RM, Alexander GC. Measuring satisfaction with amplification in daily life: the SADL scale. Ear Hear. 1999, 20(4):306-20.

11. Veiga LR. Investigação sobre a satisfação com a prótese auditiva na vida diária em usuários do sistema de saúde do exército [dissertação]. Porto Alegre: Universidade Federal do Rio Grande do Sul - Faculdade de Medicina; 2004.

12. Veiga LR, Merlo ARC, Mengue SS. Satisfação com a prótese auditiva na vida diária em usuários do sistema de saúde do exército. Rev Bras Otorrinolaringol. 2005, 71(1):67-73.

13. Hosford-Dunn H, Halpern J. Clinical application of the satisfaction with amplification in daily life scale in private practice I: statistical, content and factorial validity. J Am Acad Audiol. 2000, 11(10):523-39.

14. Northern JL. Pacient satisfaction and hearing aid outcomes. The Hearing Journal. 2000, 53(6):10-16.

15. CarvalhoJSA. Satisfação de idosos com aparelhos auditivos concedidos no estado do Tocantins. Arq Int Otorrinolaringol. 2007, 11(4):416-26.

16. Soares DO, Tavares RA, Ferreira RT, Guglielmino G, Dinato C, Franchi VM. Satisfação dos usuários de prótese auditiva em seu dia-a-dia. ACTA ORL. 2007, 25(4):290-92.

17. Weinstein B. Treatment efficacy: hearing aids in the management of hearing loss in adults. Journal of Speech and Hearing Research. 1996, 39(5):S37-S45. 
18. Magni C, Freiberger F, Tonn K. Avaliação do grau de satisfação entre os usuários de amplificação de tecnologia analógica e digital. Rev Bras Otorrinolaringol. 2005, 71(5):650-57.

19. Teixeira CF, Augusto LGS, Caldas Neto SS. Prótese auditiva: satisfação do usuário com sua prótese e com seu meio ambiente. Rev CEFAC. 2008, 10(2):245-53.

20. Megighian D, Savastano M, Salvador L, Frigo A, Bolzan M. Audiometric and epidemiological analysis of elderly in the veneto region. Gerontology. 2000, 46(4):199-204.

21. Helfer KS. Gender, age and hearing. Seminars in Hearing. 2001, 22(3):271-86.
22. Courtenay WH. Constructions of masculinity and their influence on mens well-being: a theory of gender and health Soc Sci Med. 2000, 50(10): 1385-1401.

23. Figueiredo W. Assistência à saúde dos homens: um desafio para os serviços de atenção primária. Cien Saude Colet. 2005, 10(1):105-109.

24. Costa-Junior FM, Maia ACB. Concepções de homens hospitalizados sobre a relação entre gênero e saúde. Psic.: Teor. e Pesq. 2009, 25(1):055-063. 